\title{
Successful treatment of metastatic colorectal cancer with apatinib: report of two cases and literature review
}

This article was published in the following Dove Press journal: OncoTargets and Therapy

\section{Minghong Bi* \\ Jingru Yang* \\ Yaping Wang \\ Haoran Zhang \\ Zhenyuan Gao \\ Hairong Zhou \\ Mohan Shi}

Department of Medical Oncology, The First Affiliated Hospital of Bengbu

Medical College, Bengbu, Anhui

Province, People's Republic of China

*These authors contributed equally to this work

\section{Video abstract}

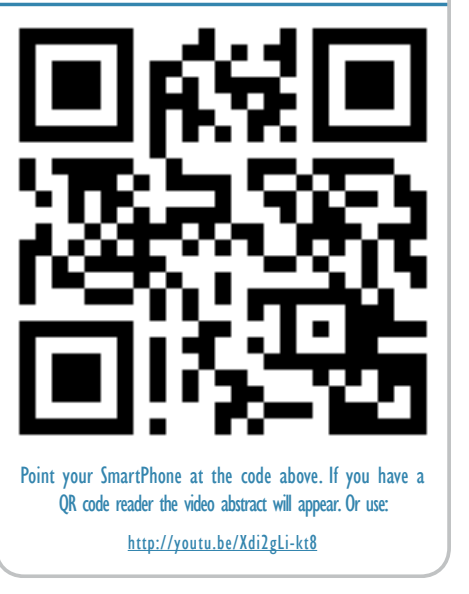

Correspondence: Minghong $\mathrm{Bi}$ Department of Medical Oncology, The First Affiliated Hospital of Bengbu Medical College, No 287 Changhuai Road, Bengbu 233000, Anhui Province, People's Republic of China

$\mathrm{Tel}+86$ I38 552। 5I68

Email ahbmh2000@I63.com

\begin{abstract}
Apatinib, a novel small molecule tyrosine kinase inhibitor that inhibits vascular endothelial growth factor receptor-2, was approved for metastatic gastric adenocarcinoma in China in Oct 2014. This is the first report on its use for advanced colorectal cancer as a kind of third-line therapy to date. Here we report two Chinese patients who presented with metastatic colorectal cancer who received apatinib $850 \mathrm{mg}$ daily as a third-line therapy. Both the patients achieved favorable benefits in outcomes after the administration of apatinib. Patient 1 benefited 4 months progression-free survival and 11 months overall survival, while patient 2's progression-free survival was over 10 months. Both the patients presented hand-foot syndrome, and one of them suffered a slight impairment of liver function, mild elevated blood pressure, and proteinuria. But these adverse events were manageable with symptomatic treatment and dose reduction or a short-time drug withdrawal.
\end{abstract}

Keywords: vascular endothelial growth factor, large intestine cancer, apatinib, targeted therapy, antiangiogenesis, tyrosine kinase inhibitor

\section{Introduction}

Colorectal cancer (CRC) was the fifth most frequently diagnosed cancer and leading cause of cancer mortality in China in $2015 .{ }^{1}$ Approximately $20 \%$ of patients who are initially diagnosed with CRC are metastatic, and almost $50 \%$ of patients who initially presented with early stage CRC will finally develop metastases. ${ }^{2}$ Despite rapid progress in diagnostic and therapeutic techniques, the 5-year survival rate of patients with metastatic CRC (mCRC) is just about $11 \%{ }^{3}$ Since the approval of bevacizumab in 2004, antiangiogenesis agents targeting vascular endothelial growth factor (VEGF) pathway have been of particular interest in treating $\mathrm{mCRC}$. Several new antiangiogenic agents, such as aflibercept, regorafenib, and ramucirumab, have also shown clinical benefits in some of the recent large Phase III studies, with an improvement of median overall survival (OS) to 30-33 months in advanced CRC patients. ${ }^{4,5}$

Apatinib, a novel small-molecule tyrosine kinase inhibitor (TKI) selectively inhibiting VEGF receptor-2 (VEGFR-2), thus preventing angiogenesis, is indicated for treating advanced gastric cancer as a third-line therapy in China.${ }^{6}$ Given its favorable safety profile and clinical efficacy, apatinib has demonstrated a substantial potential in treating a wide range of tumor settings including lung, colon, breast, and hepatocellular cancers. ${ }^{7}$ So far, there has been no literature report of using apatinib for treating mCRC. In this paper, we report two advanced mCRC patients who were treated with apatinib as a third-line therapy. 


\section{Case report I}

Patient 1, a 52-year-old female, was admitted to our hospital in Jun 2014, due to "blood-stained stool for 2 years". Colonoscopy revealed a mass in rectum, and preoperative pathological diagnosis was adenocarcinoma. On July, 2014, the loop sigmoid colostomy was performed, which revealed about 300 $\mathrm{mL}$ bloody ascites in pelvis. A $9 \times 8 \times 6 \mathrm{~cm}$ mass was found in segment IV of liver, and some pale nodules were distributed in the abdominal cavity and right ovary, which were diagnosed as pelvic mucinous adenocarcinoma planting metastasis (by intraoperative frozen section examination). The diagnosis was rectal adenocarcinoma with diffused pelvic and liver metastasis (T4N2M1 stage IV). No gene mutations were detected in $R A S$ and $B R A F$. Because our department was conducting clinical research comparing the efficacy of raltitrexed versus fluoropyrimidine in treating $\mathrm{mCRC}$ at that time when the patient was admitted to our hospital, we enrolled the patient in our research and administered the patient "oxaliplatin + raltitrexed” for three cycles (Jul 2014 to Sep 2014). Unfortunately, her follow-up computed tomography (CT) scans indicated progressive disease (PD). Concerning the patient's poor response, and severe gastrointestinal symptoms, we recommended the patient use $\mathrm{S}-1$, which is an oral $5-\mathrm{FU}$ prodrug that has slight gastrointestinal toxicity. Her treatment was then altered for three cycles of "oxaliplatin + S-1" regimen, as the second-line chemotherapy (Oct 2014-Dec 2014). The therapeutic evaluation was stable disease (SD). Then, she was administered S-1 orally as a maintenance treatment for six cycles (Jan 2015 to Jun 2015) until the patient obtained PD on CT scans. The patient's condition deteriorated with increased ascites and aggravated gastrointestinal symptoms. Afterward, "Cisplatin + Endostatin" hyperthermia peritoneal perfusion chemotherapy was applied three times to treat massive ascites in Jul 2015. The patient obtained PD on CT scans after the termination of chemotherapy. Trifluridine/ tipiracil (TAS-102) and regorafenib were not approved in China at that time, and there were no suitable clinical trials of mCRC in our hospital. The patient refused to consult in other hospitals or purchase expensive targeted agents. Apatinib went on the market at that time with a favorable policy for purchasing. After signing informed consent, the patient started taking apatinib $(850 \mathrm{mg} /$ day $)$ in Oct 2015. Four months later, the follow-up CT scan indicated an SD by Response Evaluation Criteria in Solid Tumors (RECIST 1.1) with a $22.2 \%$ decrease of liver target lesions compared with the baseline of Oct 2015 (Figure 1). However, due to severe abdominal distension and loss of appetite, we had to terminate the use of apatinib, and we gave the patient immediate and appropriate care in Mar 2016. The patient finally died of terminal CRC on Sep 25, 2016; the OS was 11 months. The treatment timeline of the patient is presented in Figure 2. Because of the side effects of hand-foot syndrome (HFS) (grade 2) and proteinuria (grade 2), the patient had a shorttime drug withdrawal, and recovered from the side effects with symptomatic treatment during apatinib treatment.

\section{Case report 2}

Patient 2, a 59-year-old man, who suffered blood-stained stool for more than 2 months, underwent colonoscopy in our hospital in Mar 2013. Histopathological detection revealed a rectal adenocarcinoma. On Mar 8, 2013, he underwent Dixon's operation. Histopathology examination revealed rectum adenocarcinoma with high-grade dysplasia (with a measured area of $5 \times 4 \times 1.2 \mathrm{~cm}$ ) invading all layers of the bowel wall and surrounding nerves, but free of tumor cells at the cutting edge. No gene mutations were detected in $R A S$ and $B R A F$. The patient
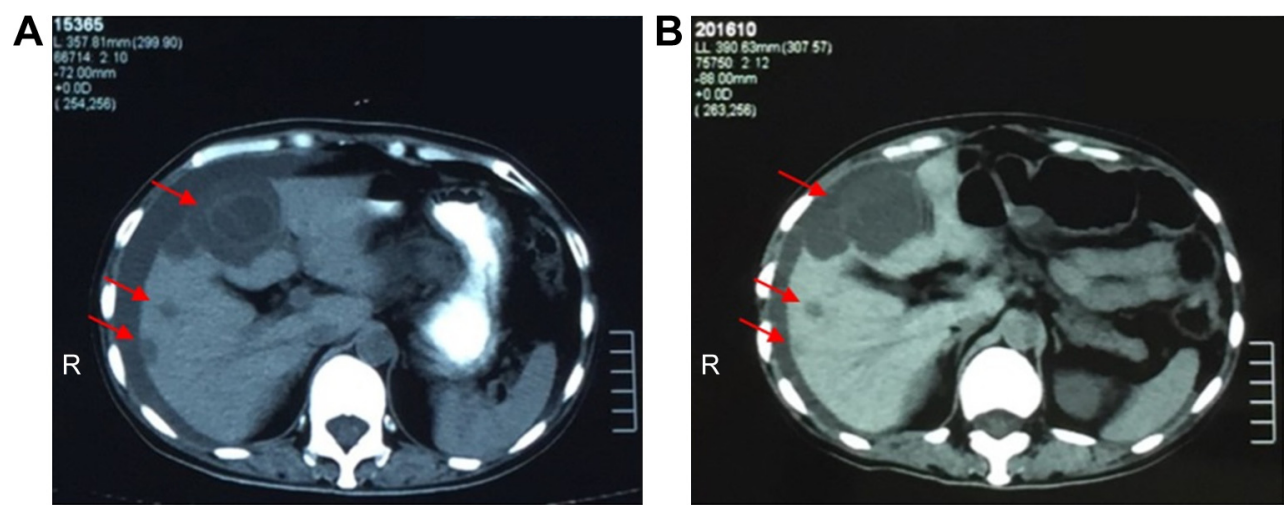

Figure I Patient I's response to apatinib.

Notes: (A) CT scan shows that liver metastasis lesions are low in density and irregular with ascites prior to the use of apatinib (Oct 2015). (B) After 4 months of apatinib treatment, decrease in the lesions and smaller amount of ascites were seen (Jan 2016).

Abbreviation: CT, computed tomography. 


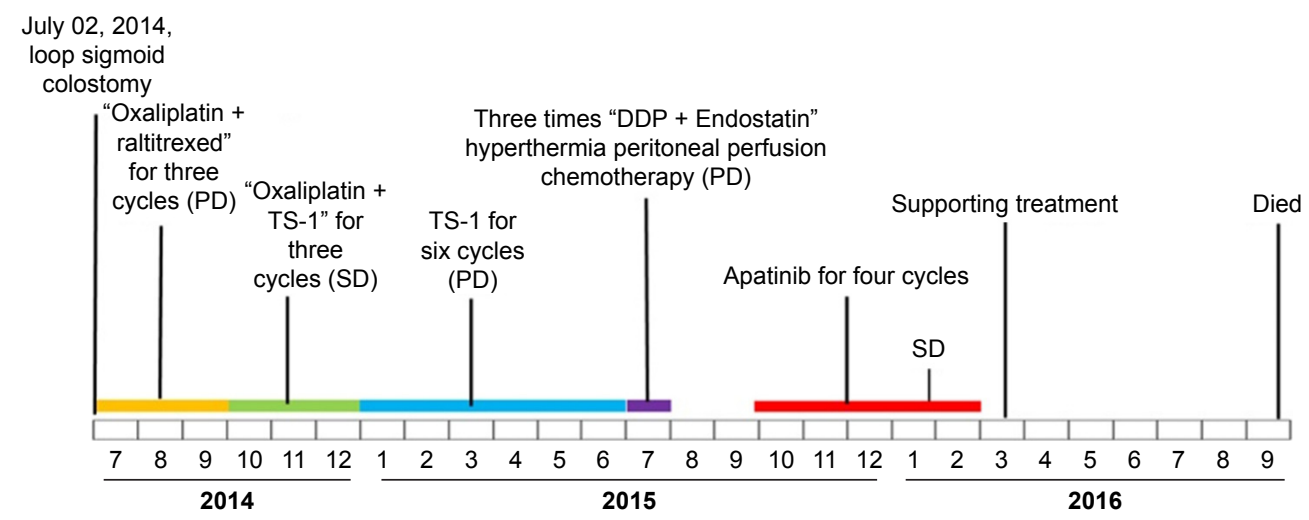

Figure 2 Timeline of Patient I's management.

Abbreviations: DDP, Cisplatin; PD, progressive disease; SD, stable disease.

had a magnetic resonance (MR) imaging on Mar 21, 2013, due to lumbosacral pain, and it demonstrated abnormal signal intensity in the sacral vertebrae. The diagnosis was stage IV rectal adenocarcinoma with pelvic metastases (T4N1M1). In Apr 2013, in a local hospital, the patient underwent Gamma Knife Surgery for the rectum and pelvic lesions with a dose of $260 \mathrm{cGy}$ at the $50 \%$ isodose margin. Unfortunately, he refused to receive concurrent chemotherapy out of his own reasons. We gave him sequential chemotherapy with FOLFOX6 regimen (leucovorin, fluorouracil, and oxaliplatin) at the end of radiotherapy for four cycles (May 2013-Aug 2013). The efficacy was evaluated as partial response. From Aug 2013 to Jun 2015, almost 2 years, the tumor was relatively stable without apparent progression and therapy. In Jul 2015, he presented with severe abdominal distension. His abdominal ultrasonography and CT scans revealed a space occupation in liver, which indicated the recurrence of the tumor with liver metastasis. Then, the patient received one cycle of second-line chemotherapy - FOLFIRI consisting of irinotecan, fluorouracil, and leucovorin. Significant diarrhea occurred and the patient refused further chemotherapy. After providing written, informed consent, the patient started oral administration of apatinib $850 \mathrm{mg}$ once a day from Aug 12,
2015. Compared with the baseline of Jul 2015, CT scan indicated a $27.7 \%$ decrease in the longest diameters of the target liver lesion after administering apatinib for 3 months. The therapeutic evaluation was SD by RECIST 1.1. After 10 months of apatinib administration, CT scan revealed an SD by RECIST with a 7.6\% decrease in the longest diameters of target liver lesion (Figure 3). He maintained his stable radiological response for further 10 months until progression in the size of the tumor was demonstrated on repeat CT in Sep 2016, which evaluated progressive disease (PD), and we stopped the application of apatinib. Until now, the patient is still alive with best supportive treatment (date locked in Jun 2017). The treatment timeline of the patient is presented in Figure 4. During the period of apatinib therapy, the patient encountered grade 3 HFSS at the first month of apatinib therapy. It was manageable with symptomatic treatment and did not necessitate treatment discontinuation. The patient also experienced mild elevated blood pressure (grade 2), proteinuria (grade 1), slightly elevated transaminase, and bilirubin (grade 1) later. We gave him antihypertensive and liver-protection drugs and reduced the dose of apatinib to $425 \mathrm{mg}$ daily as a maintenance therapy. These adverse effects were well controlled and tolerated.
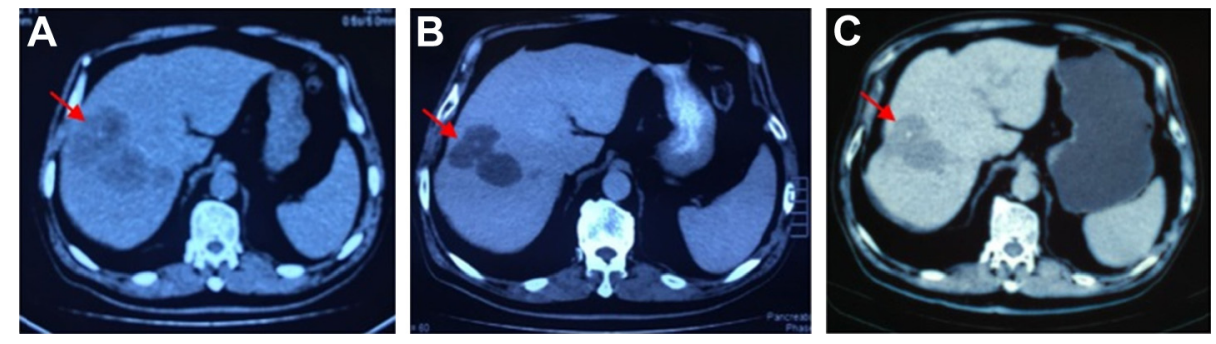

Figure 3 Patient 2's response to apatinib.

Notes: (A) CT scans show the liver metastasis lesion prior to apatinib (Jul 20I5). (B) After 4 months of apatinib therapy (Nov 20I5), CT scans show that the size of the metastasis lesion was decreased. (C) After 10 months of apatinib therapy (Jun 2016), the lesion showed a sustained reduction in size. Abbreviation: CT, computed tomography. 


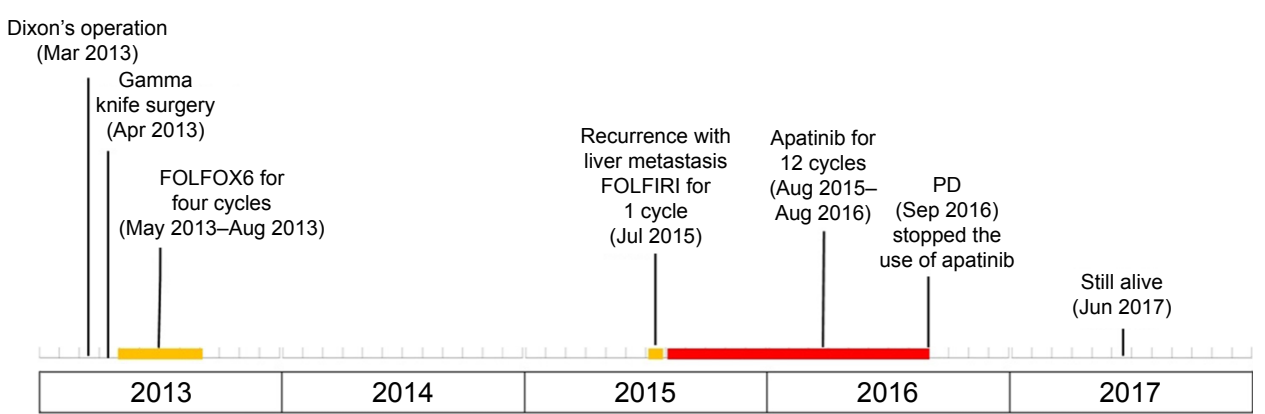

Figure 4 Timeline of Patient 2's management.

Abbreviation: PD, progressive disease.

\section{Discussion}

The standard regimens used in late-stage $\mathrm{mCRC}$ are based on fluoropyrimidines combined with oxaliplatin or irinotecan and with or without one molecularly targeted drug including antiestimated glomerular filtration rate (EGFR) monoclonal antibody (eg, cetuximab, panitumumab, only being limited to treat patients with $K R A S / N R A S$ wide-type left semicolon tumors), antiangiogenic inhibitors (eg, bevacizumab, aflibercept, and ramucirumab), multiple receptor TKI (regorafenib), or PD-1 inhibitors (nivolumab or pembrolizumab, in dMMR/MSI-H only). Single-agent regorafenib or trifluridine + tipiracil is also recommended on second-line or above therapy. ${ }^{8}$

Neovascularization (sprouting angiogenesis) plays a pivotal role in the proliferation, development, and progression in multiple malignancies; large amount of blood vessels can supply the necessary oxygen and nutrients and dispose of catabolic products. ${ }^{9}$ VEGF is regarded as one kind of crucial regulator in proangiogenesis, which includes VEGF-A, -B, $-\mathrm{C},-\mathrm{D},-\mathrm{E}$, and placental growth factor (PlGF). Binding of VEGF to its tyrosine kinase receptors, VEGFR1-3 located in tumor-associated endothelial cells, triggers a cascade of multiple signaling pathways including RAS/MAPK, PI3KAKT, PKC, and FAK downstream pathways, thus leading to endothelial cell proliferation, new blood vessels generation, thereby stimulating the tumor growth and migration. ${ }^{10}$ VEGFs-VEGFRs axis is often overexpressed in a wide spectrum of carcinomas, and inhibiting VEGF pathway is the benchmark in developing novel antiangiogenic inhibitors in recent decades. Currently, multiple antiangiogenic agents targeting VEGFs-VEGFRs and multitarget agents containing antiangiogenic and antiproliferative effects have been approved in clinical treatments. ${ }^{11}$ Antiangiogenetic agents targeting the VEGF system also play a crucial role in treating $\mathrm{mCRC}$, especially in improving OS. Bevacizumab, aflibercept, regorafenib, and recently ramucirumab have been demonstrated to be effective with a manageable toxicity profile and are now approved by regulatory agencies. ${ }^{12}$
Bevacizumab is a recombinant monoclonal antibody with a high VEGF-neutralizing specificity, preventing the binding of VEGF-A to VEGFR-1 and VEGFR-2. ${ }^{13}$ Landmark AVF2107 trial has proven that the addition of bevacizumab to irinotecan, bolus fluorouracil, and leucovorin (IFL) regimen significantly improved progression-free survival (PFS; 10.6 vs 6.2 months, hazard ratio [HR] $=0.54, P<0.001)$, OS (20.3 vs 15.6 months; $\mathrm{HR}=0.66, P<0.001$ ), and objective response rate (ORR; $44.8 \%$ vs $34.8 \%, P=0.004),{ }^{14}$ thus leading to the approval of bevacizumab in treating $\mathrm{mCRC}$ in first-line setting in 2004. Several Phase III trials, ML 18147, BEBYP, and EAGLE, also indicated that continued bevacizumab beyond the first-line treatment statistically prolongs OS, although with a very modest benefit in mCRC patients. ${ }^{15-17}$ The ECOG E3200 study described an OS benefit when bevacizumab was added to second-line chemotherapy in mCRC patients who progressed after first-line non-bevacizumab-containing setting..$^{13}$ Moreover, the large-scale trials CAIRO3, AIO 0207, SAKK $41 / 06,{ }^{18}$ and OPTIMOX3 consensually concluded a considerable delay of tumor progression with an HR of about 0.45 , while on maintenance treatment containing bevacizumab combined with a fluoropyrimidine. ${ }^{19-21}$

Aflibercept (VEGF-trap, zip-Aflibercept) is a novel type of human recombinant fusion protein containing the VEGF receptor 1 and 2 domains, acting as a high-affinity ligand trap by preventing the interaction of VEGF-A, VEGF-B, and PIGF with their extracellular receptors, with a wider spectrum of targets compared with bevacizumab. ${ }^{22}$ In Phase III VELOUR trial, 1,226 of all the enrolled CRC patients who progressed after first-line oxaliplatin-containing therapy showed that FOLFIRI in combination with aflibercept compared with FOLFIRI plus placebo improved OS (13.5 vs 12.06 months, HR 0.817; $P=0.0032$ ), PFS (6.9 vs 4.67 months, HR 0.758; $P<0.0001)$, and ORR (19.8\% vs $11.1 \% ; P=0.0001)$, but a greater incidence of adverse events (AEs) in aflibercept arm was reported. ${ }^{23}$ Based on these data, in 2012, the US Food and Drug Administration (FDA) approved aflibercept combined 
with FOLFIRI or irinotecan as a second-line option in mCRC patients progressed or being resistant after a first-line chemotherapy not including irinotecan. ${ }^{22}$

Regorafenib is an oral multi-TKI inhibiting various protein kinases, including antiangiogenesis (VEGFR1/3, TIE2), antioncogenesis (KIT, RET, RAF-1, B-RAF), and antistromal (PDGFR- $\alpha$, PDGFR- $\beta$, FGFR) properties. ${ }^{24}$ The Phase III CORRECT trial showed that in treatment-refractory mCRC patients, single-agent regorafenib prolonged its primary endpoints with an OS of 6.4 vs 5.0 months (HR 0.77 ; $P=0.0052$ ), and the PFS also improved of 1.9 vs 1.7 months (HR 0.49; $P<0.000001$ ). The CONCUR trial also described similar benefits in Asian mCRC patients. Currently, regorafenib is indicated in refractory $\mathrm{mCRC}$ patients to standard chemotherapy as an additional line of treatment. ${ }^{25,26}$

Ramucirumab, a fully human IgG1 monoclonal antibody selectively targeting VEGFR-2 and thus blocking the VEGF signal pathways, has been approved for treating several malignancy indications. ${ }^{27}$ In Apr 2015, the FDA approved ramucirumab in conjunction with FOLFIRI or irinotecan as a second-line option for $\mathrm{mCRC}$ patients who had failed in firstline therapy not including irinotecan. This approval was based on RAISE trial which showed that ramucirumab plus FOLFIRI improved both the OS (13.3 vs 11.7 months, $P=0.0219)$ and PFS (5.7 vs 4.5 months, $P<0.0005$ ) compared with FOLFIRI alone in $\mathrm{mCRC}$ patients who have failed with first-line oxaliplatin/fluoropyrimidines/bevacizumab-based chemotherapy. ${ }^{28}$

Apatinib (YN968D1) is a small-molecule TKI that selectively binds to and potently suppresses the kinase activities of VEGFR-2 and also moderately suppresses RET, c-kit, c-SRC, and has effects on EGFR, HER-2, FGFR, thus decreasing VEGF-mediated endothelial cell migration, proliferation, and tumor microvascular density. VEGFR-2 is identified as one kind of crucial regulator of the whole process of angiogenesis. Activation of VEGFR-2, mediated by VEGFs, plays a preferential role in triggering the cascade of proangiogenetic signaling pathways with respect to other VEGFRs. In preclinical models, apatinib was active against a wide spectrum of malignancies, including gastric, lung, hepatocellular, breast, and colorectal cancers. ${ }^{29,30}$ In a Phase III study, in patients with chemotherapy-refractory advanced metastatic gastric cancer, compared with placebo, apatinib has shown exciting effects and good safety profile, with an mOS of 195 vs 140 days $(P=0.0156)$, mPFS of 78 vs 53 days $(P<0.001)$, ORR of $2.8 \%$ vs $0 \%(P=0.1695)$, and disease control rate (DCR) of $42.1 \%$ vs $8.8 \%(P<0.001) .{ }^{31}$ In 2014, China FDA approved apatinib as a third-line therapy for metastatic gastric cancer. ${ }^{32}$ Currently, apatinib is being investigated in different tumor settings (including breast, lung, hepatocellular, colorectal, biliary tract, ovarian, pancreatic, head and neck cancer, osteosarcoma, etc.), different chemotherapy regimens (such as capecitabine plus oxaliplatin $[\mathrm{XELOX}]$ to $\mathrm{CRC}, \mathrm{S}-1$ or pemetrexed or etoposide or docetaxel to non-small cell lung carcinoma [NSCLC], S-1 plus oxaliplatin $[\mathrm{SOX}]$ or oxaliplatin or $\mathrm{S}-1$ to gastric cancer, vinorelbine to breast cancer, etoposide to ovarian cancer), and different targeted drugs (gefitinib to EGFR mutant NSCLC [NCT02824458], SHR-1210 to hepatocellular or gastric cancer [NCT02942329], etc.). In in vivo models, apatinib significantly inhibited the growth of human colon tumor xenograft, the tumor growth inhibitory rate being $40 \%$ $81.2 \%$ with good tolerance. In addition, the combination treatment of apatinib plus oxaliplatin or 5-FU also showed a successful antitumor efficacy, the tumor inhibition rate being $60.1 \%$ and $58.6 \%$, respectively. ${ }^{29}$ One preclinical trial also found that apatinib can significantly inhibit the proliferation, migration, and invasion of colon cancer cells, and it was involved in the regulation of autophagy.$^{33}$ In addition, another preclinical trial used Patient-derived xenograft (PDX) model of KRAS-mutant CRC and showed that apatinib combined with irinotecan (CPT-11) treatment significantly inhibited the growth of tumors, reduced microvessel density, suppressed proliferation, and increased apoptosis, which suggested a rational combination strategy of apatinib and CPT-11 for patients with $K R A S$-mutant CRC. ${ }^{34}$ In a Phase II trial of apatinib in refractory mCRC patients (NCT01531777), ${ }^{35}$ totally, 40 advanced or metastatic CRC patients who failed over two lines of treatment were randomized to accept apatinib $750 \mathrm{mg} /$ day or $500 \mathrm{mg} /$ day. Patients continued the usage of apatinib till disease progression, intolerable toxicity, or consent withdrawal occurred. The trial has recently been completed, and we are waiting for the final results. Recently, in another Phase II trial, apatinib (500 mg, oral, days 1-21) combined with XELOX (oxaliplatin $130 \mathrm{mg} / \mathrm{m}^{2}$ intravenous injection day 1 , capecitabine $1,000 \mathrm{mg}$ oral days $1-14$ ) has been assigned to treat end-stage CRC patients as a first-line therapy. The treatment cycle is every three weeks till PD or intolerance of drug toxicity occurs. The trial is recruiting participants (NCT02829385). Apatinib has shown significant efficacy in treating $\mathrm{mCRC}$, and follow-up research on this is in progress. However, since sampling is mostly small, relevant studies are relatively limited. More research is needed to provide high-stage clinical evidence to guide clinical usage of apatinib.

The two cases in our study failed in second-line chemotherapy and were no longer able to tolerate further chemotherapy. Several target agents mentioned above are recommended in treating advanced or metastatic CRC. 
However, using these drugs is limited by a series of factors, including the difficulty in accessibility, availability, and high cost, especially in developing countries. Several anti-VEGF drugs are recommended to treat advanced CRC in National Comprehensive Cancer Network Guidelines. Similarly, we speculated that apatinib may have a potential efficacy profile by selectively inhibiting VEGFR-2. So, we provided apatinib to both patients at a dose of $850 \mathrm{mg}$ as tablet daily. After the period of therapy, patient 1 showed 4 months of PFS, and patient 2 gained significant efficacy with 10 months of PFS. The different outcomes of the two patients indicated that the clinical efficacy may be different in individuals because of heterogeneity of the tumor and may also be associated with the stage of the tumor and the patients' general conditions. Further studies need to be conducted to identify suitable biomarkers to predict drug efficacy, which would reduce the blindness in selecting suitable patients and improve efficacy. Occurrence of treatment-related side effects such as hypertension, proteinuria, and hand-foot skin reaction (HFSR) might be predictors of prolonged PFS and OS as one kind of pharmacodynamics effects of antiangiogenic drugs. Higher expression of phosphorylated VEGFR-2 is also related to the clinical benefits of VEGF inhibitors. ${ }^{36,37}$ Further studies need to be conducted to validate predictive or prognostic factors for antiangiogenic agents.

Both in Phase II and III trials of apatinib, the most common AEs of apatinib are hypertension, proteinuria, and HFSS. They are associated with nontarget effects, and grade 3 and 4 AEs occur in about $2 \%$ of the patients. Other minor AEs include fatigue, thrombocytopenia, liver function impairment (elevated aminotransferase and bilirubin), and diarrhea. All these apatinib-related toxicities are relevant to antiangiogenics, as occurring in other VEGF/VEGFR inhibitors, and no new AEs have been found in apatinib..$^{6,31,38,39}$ Both the cases in our study showed HFSS which was relieved by temporary dose interruption and symptomatic therapy, with no influence on the further therapy. The mechanism of HFSS may be the hands and feet are rich in capillaries, and angiogenesis inhibitors could degradate the vascular bed, thus leading to the skin damage. ${ }^{40}$

\section{Conclusion}

In summary, the response to apatinib from our patients showed that apatinib may prolong the survival of patients with mCRC with a good safety and tolerability profile. Nevertheless, there are still some questions that require further investigation. First, apatinib has only been proven to be safe and active in a subset of gastric cancer. Questions remain as to whether it is applicable in other types of tumors; if suitable, in first-, second-, or third-line therapy; and if it should be taken as a single agent or combined with other therapies? Second, the current apatinib-relevant clinical trials were conducted in China, and the efficacy may be influenced by regional difference, which calls for following research on the efficacy in other ethnic groups. Last but most important, the possible mechanisms of drug resistance, alternative triggers of angiogenesis, and the potential predictive biomarkers that could help to better select patients are still unknown. All these questions call for more large-scale, global, multicenter clinical studies and investigations.

\section{Acknowledgments}

This study was supported in part by The Research Project of Anhui Provincial Health Bureau (09C171), Science and Technology Development Fund of Bengbu Medical College (BYKF1403), and the Universities Natural Science Research Project in Anhui Province (KJ2016A483). Written informed consent has been provided by the two patients to have the case details and any accompanying images published. The authors thank the two patients for participation, and the contributions of all in the treatment group in the Department of Medical Oncology.

\section{Disclosure}

The first authors of this manuscript are Minghong Bi and Jingru Yang. The authors report no conflicts of interest in this work.

\section{References}

1. Chen W, Zheng R, Baade PD, et al. Cancer statistics in China, 2015. CA Cancer J Clin. 2016;66(2):115-132.

2. Van Cutsem E, Cervantes A, Nordlinger B, Arnold D; ESMO Guidelines Working Group. Metastatic colorectal cancer: ESMO Clinical Practice Guidelines for diagnosis, treatment and follow-up. Ann Oncol. 2014;25(Suppl 3):iii1-iii9.

3. Scudellari M. Drug development: Mix and match. Nature. 2015; 521(7551):S12-S14.

4. Heinemann V, von Weikersthal LF, Decker T, et al. FOLFIRI plus cetuximab versus FOLFIRI plus bevacizumab as first-line treatment for patients with metastatic colorectal cancer (FIRE-3): a randomised, open-label, phase 3 trial. Lancet Oncol. 2014;15(10):1065-1075.

5. Venook A, Niedzwiecki D, Lenz HJ, et al. O-0019CALGB/SWOG 80405: Phase III trial of irinotecan/5-FU/leucovorin (FOLFIRI) or oxaliplatin/5-FU/leucovorin (mFOLFOX6) with bevacizumab (BV) or cetuximab (CET) for patients (PTS) with KRAS wild-type (WT) untreated metastatic adenocarcinoma of the colon or rectum (MCRC). Ann Oncol. 2014;25(Suppl 2):ii112-ii113.

6. Geng R, Li J. Apatinib for the treatment of gastric cancer. Expert Opin Pharmacother. 2015;16(1):117-122.

7. Scott AJ, Messersmith WA, Jimeno A. Apatinib: a promising oral antiangiogenic agent in the treatment of multiple solid tumors. Drugs Today (Barc). 2015;51(4):223-229.

8. Benson AB 3rd, Venook AP, Cederquist L, et al. Colon cancer, version 1. 2017, NCCN clinical practice guidelines in oncology. 2017;15(3): 370-398.

9. Chung AS, Lee J, Ferrara N. Targeting the tumour vasculature: insights from physiological angiogenesis. Nat Rev Cancer. 2010;10(7):505-514. 
10. Hicklin DJ, Ellis LM. Role of the vascular endothelial growth factor pathway in tumor growth and angiogenesis. J Clin Oncol. 2005;23(5): 1011-1027.

11. Limaverde-Sousa G, Sternberg C, Ferreira CG. Antiangiogenesis beyond VEGF inhibition: a journey from antiangiogenic single-target to broad-spectrum agents. Cancer Treat Rev. 2014;40(4):548-557.

12. Khan K, Cunningham D, Chau I. Targeting angiogenic pathways in colorectal cancer: complexities, challenges and future directions. Curr Drug Targets. 2017;18(1):56-71.

13. Giantonio BJ, Catalano PJ, Meropol NJ, et al. Bevacizumab in combination with oxaliplatin, fluorouracil, and leucovorin (FOLFOX4) for previously treated metastatic colorectal cancer: results from the Eastern Cooperative Oncology Group Study E3200. J Clin Oncol. 2007;25(12):1539-1544.

14. Hurwitz H, Fehrenbacher L, Novotny W, et al. Bevacizumab plus irinotecan, fluorouracil, and leucovorin for metastatic colorectal cancer. N Engl J Med. 2004;350(23):2335-2342.

15. Bennouna J, Sastre J, Arnold D, et al; ML18147 Study Investigators. Continuation of bevacizumab after first progression in metastatic colorectal cancer (ML18147): a randomised phase 3 trial. Lancet Oncol. 2013;14(1):29-37.

16. Masi G, Salvatore L, Boni L, et al; BEBYP Study Investigators. Continuation or reintroduction of bevacizumab beyond progression to first-line therapy in metastatic colorectal cancer: final results of the randomized BEBYP trial. Ann Oncol. 2015;26(4):724-730.

17. Iwamoto $\mathrm{S}$, Takahashi $\mathrm{T}$, Tamagawa $\mathrm{H}$, et al. FOLFIRI plus bevacizumab as second-line therapy in patients with metastatic colorectal cancer after first-line bevacizumab plus oxaliplatin-based therapy: the randomized phase III EAGLE study. Ann Oncol. 2015;26(7):1427-1433.

18. Koeberle D, Betticher DC, von Moos R, et al. Bevacizumab continuation versus no continuation after first-line chemotherapy plus bevacizumab in patients with metastatic colorectal cancer: a randomized phase III non-inferiority trial (SAKK 41/06). Annals of oncology: official journal of the European Society for Medical Oncology / ESMO. Apr 2015;26(4):709-714.

19. Simkens LH, van Tinteren H, May A, et al. Maintenance treatment with capecitabine and bevacizumab in metastatic colorectal cancer (CAIRO3): a phase 3 randomised controlled trial of the Dutch Colorectal Cancer Group. Lancet. 2015;385(9980):1843-1852.

20. Hegewisch-Becker S, Graeven U, Lerchenmuller CA, et al. Maintenance strategies after first-line oxaliplatin plus fluoropyrimidine plus bevacizumab for patients with metastatic colorectal cancer (AIO 0207): a randomised, non-inferiority, open-label, phase 3 trial. Lancet Oncol. 2015;16(13):1355-1369.

21. Tournigand C, Chibaudel B, Samson B, et al. Bevacizumab with or without erlotinib as maintenance therapy in patients with metastatic colorectal cancer (GERCOR DREAM; OPTIMOX3): a randomised, open-label, phase 3 trial. Lancet Oncol. 2015;16(15):1493-1505.

22. Ciombor KK, Berlin J. Aflibercept-a decoy VEGF receptor. Curr Oncol Rep. 2014;16(2):368.

23. Van Cutsem E, Tabernero J, Lakomy R, et al. Addition of aflibercept to fluorouracil, leucovorin, and irinotecan improves survival in a phase III randomized trial in patients with metastatic colorectal cancer previously treated with an oxaliplatin-based regimen. J Clin Oncol. 2012;30(28):3499-3506.

24. Wilhelm SM, Dumas J, Adnane L, et al. Regorafenib (BAY 73-4506): a new oral multikinase inhibitor of angiogenic, stromal and oncogenic receptor tyrosine kinases with potent preclinical antitumor activity. Int J Cancer. 2011;129(1):245-255.
25. Grothey A, Van Cutsem E, Sobrero A, et al; CORRECT Study Group. Regorafenib monotherapy for previously treated metastatic colorectal cancer (CORRECT): an international, multicentre, randomised, placebo-controlled, phase 3 trial. Lancet. 2013;381(9863):303-312.

26. Li J, Qin S, Xu R, et al; CONCUR Investigators. Regorafenib plus best supportive care versus placebo plus best supportive care in Asian patients with previously treated metastatic colorectal cancer (CONCUR): a randomised, double-blind, placebo-controlled, phase 3 trial. Lancet Oncol. 2015;16(6):619-629.

27. Calvetti L, Pilotto S, Carbognin L, et al. The coming of ramucirumab in the landscape of anti-angiogenic drugs: potential clinical and translational perspectives. Expert OpinBiolTher. 2015;15(9):1359-1370.

28. Tabernero J, Yoshino T, Cohn AL, et al. Ramucirumab versus placebo in combination with second-line FOLFIRI in patients with metastatic colorectal carcinoma that progressed during or after first-line therapy with bevacizumab, oxaliplatin, and a fluoropyrimidine (RAISE): a randomised, double-blind, multicentre, phase 3 study. Lancet Oncol. 2015;16(5):499-508.

29. Tian S, Quan H, Xie C, et al. YN968D1 is a novel and selective inhibitor of vascular endothelial growth factor receptor-2 tyrosine kinase with potent activity in vitro and in vivo. Cancer Sci. 2011; 102(7):1374-1380.

30. Lu D, Jimenez X, Zhang H, Bohlen P, Witte L, Zhu Z. Selection of high affinity human neutralizing antibodies to VEGFR2 from a large antibody phage display library for antiangiogenesis therapy. Int $J$ Cancer. 2002;97(3):393-399.

31. Li J, Qin S, Xu J, et al. Randomized, double-blind, placebo-controlled Phase III trial of apatinib in patients with chemotherapy-refractory advanced or metastatic adenocarcinoma of the stomach or gastroesophageal junction. J Clin Oncol. 2016;34(13):1448-1454.

32. Aoyama T, Yoshikawa T. Targeted therapy: apatinib - new third-line option for refractory gastric or GEJ cancer. Nat Rev Clin Oncol. 2016; 13(5):268-270.

33. Lu W, Ke H, Qianshan D, Zhen W, Guoan X, Honggang Y. Apatinib has anti-tumor effects and induces autophagy in colon cancer cells. Iran J Basic Med Sci. 2017;20(9):990-995.

34. Chen X, Guan Z, Lu J, et al. Preclinical activity of the rational combination of apatinib in combination with CPT-11 in KRAS-mutant colorectal cancer patient-derived xenograft model. Int J Clin Exp Med. 2017;10(2):2274-2284.

35. Dose Finding and Pharmacokinetics/Pharmacodynamics Study of Apatinib in the Treatment of Advanced Colorectal Cancer. Available online: https://clinicaltrials.gov/ct2/show/NCT01531777.

36. Hamnvik OP, Choueiri TK, Turchin A, et al. Clinical risk factors for the development of hypertension in patients treated with inhibitors of the VEGF signaling pathway. Cancer. 2015;121(2):311-319.

37. Fan M, Zhang J, Wang Z, et al. Phosphorylated VEGFR2 and hypertension: potential biomarkers to indicate VEGF-dependency of advanced breast cancer in anti-angiogenic therapy. Breast Cancer Res Treat. 2014; 143(1):141-151

38. Li J, Zhao X, Chen L, et al. Safety and pharmacokinetics of novel selective vascular endothelial growth factor receptor-2 inhibitor YN968D1 in patients with advanced malignancies. BMC Cancer. 2010;10:529.

39. Li J, Qin S, Xu J, et al. Apatinib for chemotherapy-refractory advanced metastatic gastric cancer: results from a randomized, placebo-controlled, parallel-arm, phase II trial. J Clin Oncol. 2013;31(26):3219-3225.

40. Yu J, Zhang Y, Leung LH, Liu L, Yang F, Yao X. Efficacy and safety of angiogenesis inhibitors in advanced gastric cancer: a systematic review and meta-analysis. J Hematol Oncol. 2016;9(1):111. 


\section{Publish your work in this journal}

OncoTargets and Therapy is an international, peer-reviewed, open access journal focusing on the pathological basis of all cancers, potential targets for therapy and treatment protocols employed to improve the management of cancer patients. The journal also focuses on the impact of management programs and new therapeutic agents and protocols on

patient perspectives such as quality of life, adherence and satisfaction. The manuscript management system is completely online and includes a very quick and fair peer-review system, which is all easy to use. Visit http://www.dovepress.com/testimonials.php to read real quotes from published authors.

Submit your manuscript here: http://www.dovepress.com/oncotargets-and-therapy-journal 